\title{
Evolution and loss of long-fringed petals: a case study using a dated phylogeny of the snake gourds, Trichosanthes (Cucurbitaceae)
}

Hugo J de Boer ${ }^{1 *}$, Hanno Schaefer ${ }^{2}$, Mats Thulin ${ }^{3}$ and Susanne S Renner ${ }^{4}$

\begin{abstract}
Background: The Cucurbitaceae genus Trichosanthes comprises 90-100 species that occur from India to Japan and southeast to Australia and Fiji. Most species have large white or pale yellow petals with conspicuously fringed margins, the fringes sometimes several $\mathrm{cm}$ long. Pollination is usually by hawkmoths. Previous molecular data for a small number of species suggested that a monophyletic Trichosanthes might include the Asian genera Gymnopetalum (four species, lacking long petal fringes) and Hodgsonia (two species with petals fringed). Here we test these groups' relationships using a species sampling of c. $60 \%$ and 4759 nucleotides of nuclear and plastid DNA. To infer the time and direction of the geographic expansion of the Trichosanthes clade we employ molecular clock dating and statistical biogeographic reconstruction, and we also address the gain or loss of petal fringes.

Results: Trichosanthes is monophyletic as long as it includes Gymnopetalum, which itself is polyphyletic. The closest relative of Trichosanthes appears to be the sponge gourds, Luffa, while Hodgsonia is more distantly related. Of six morphology-based sections in Trichosanthes with more than one species, three are supported by the molecular results; two new sections appear warranted. Molecular dating and biogeographic analyses suggest an Oligocene origin of Trichosanthes in Eurasia or East Asia, followed by diversification and spread throughout the Malesian biogeographic region and into the Australian continent.

Conclusions: Long-fringed corollas evolved independently in Hodgsonia and Trichosanthes, followed by two losses in the latter coincident with shifts to other pollinators but not with long-distance dispersal events. Together with the Caribbean Linnaeosicyos, the Madagascan Ampelosicyos and the tropical African Telfairia, these cucurbit lineages represent an ideal system for more detailed studies of the evolution and function of petal fringes in plant-pollinator mutualisms.
\end{abstract}

\section{Background}

Deeply divided or fringed petal lobes are known from a range of angiosperm families, including Caryophyllaceae, Celastraceae, Cucurbitaceae, Myrtaceae, Orchidaceae, Saxifragaceae, and Tropaeolaceae [1]. While the origin and function of subdivided petals vary between groups, division of perianth edges is especially common among nocturnal hawkmoth-pollinated species (such as Trichosanthes [2], Figure 1), where the fringes, in combination with a light petal color, may enhance visibility and thus increase pollination success $[3,4]$. Experiments

\footnotetext{
* Correspondence: hugo.deboer@ebc.uu.se

'Department of Systematic Biology, Uppsala University, Norbyvägen 18 D,

Uppsala SE-75236, Sweden

Full list of author information is available at the end of the article
}

have shown that diurnal and nocturnal hawkmoths are attracted by floral scent but also rely on visual clues to find and recognize flowers even at extremely low light intensity [5,6]. A preference for high contrasts might help them find their nectar sources, and it seems plausible that fringed petals enhance the sharp contrast between the petal margin and a dark background [4].

In Cucurbitaceae, long-fringed petals are known in five genera that occur in Madagascar, tropical Africa, the Caribbean, and East and Southeast Asia [7,8]. The largest of them is Trichosanthes with currently 90-100 species of mainly perennial, 3 to $30 \mathrm{~m}$ long climbers that are usually dioecious and have medium-sized fleshy fruits. Referring to the petal fringes, Linnaeus formed the genus name from the Greek words for 'hair' (genitive 


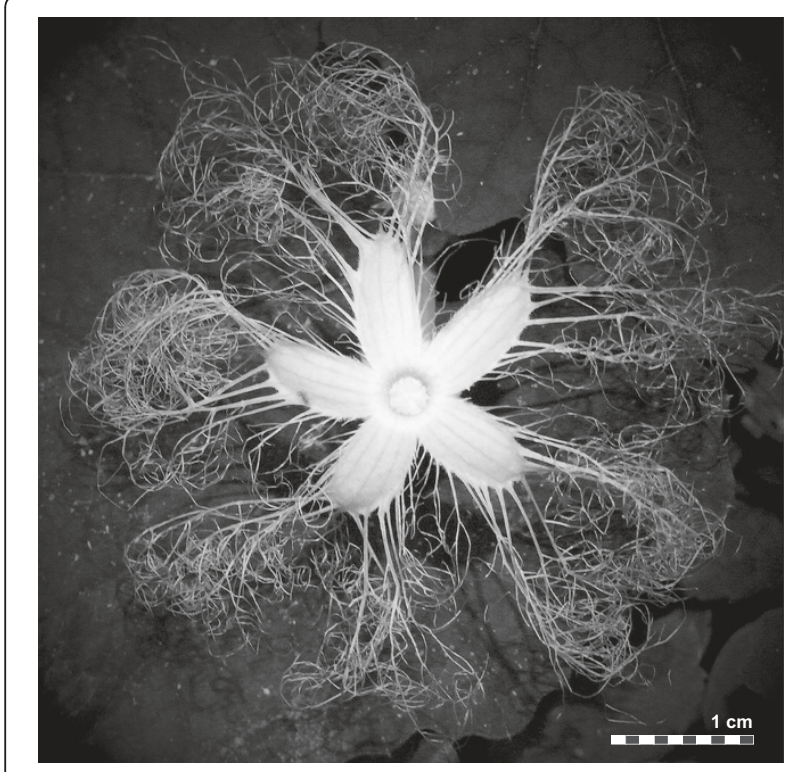

Figure 1 Fully expanded flower of Trichosanthes pilosa Lour. showing the characteristic feather-like fringes along the petal margins. Picture courtesy of Ken Ishikawa.

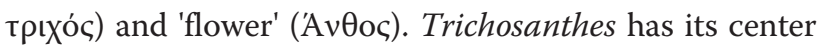
of diversity in Southeast Asia, but ranges from India throughout tropical and subtropical Asia east to Japan, and southeast to New Guinea, Australia, and Fiji [9]. One species, the snake gourd, T. cucumerina L., is a widely cultivated vegetable in tropical and subtropical regions around the globe, and another 15 species are commonly used in Asian traditional medicine [10]. While floristic treatments are available for most of its range [9,11-16], a comprehensive revision of the nearly 300 names published in Trichosanthes is lacking (but see [17] for a synopsis).

Trichosanthes belongs in the tribe Sicyoeae, a group of 12 genera and c. 270 species that is supported by morphological and molecular data [18]. Based on a limited number of Trichosanthes species sequenced, it appeared that the genus might be paraphyletic, with the genera Gymnopetalum Arn. (four species; [19]) and Hodgsonia Hook.f. \& Thomson (two species; [9]) possibly nested inside it [20]. Both share with Trichosanthes the white flowers, elongated receptacle-tubes, and free filaments. Hodgsonia also has long-fringed petals (Figure 2J), but differs from Trichosanthes and Gymnopetalum in its much larger fruits (up to $25 \mathrm{~cm}$ across) and unusual seeds. The petal margins in Gymnopetalum are entire (Figure 2A, 2E) or in one species shortly fimbriate [9]. Geographically, Gymnopetalum and Hodgsonia largely overlap with the distribution area of Trichosanthes except for their absence from New Guinea and Australia, and from much of the northeastern range of Trichosanthes (temperate China, Taiwan, Japan) [9].

Based on mainly fruit and seed characters, the 43 species of Trichosanthes occurring in the Flora Malesiana region have been grouped into six sections, the typical sect. Trichosanthes and sections Cucumeroides (Gaertn.) Kitam., Edulis Rugayah, Foliobracteola C.Y.Cheng \& Yueh, Involucraria (Ser.) Wight, and Asterosperma W.J. de Wilde \& Duyfjes [21,22]. The mainland Asian species, T. truncata C.B.Clarke, is in its own section, Truncata C.Y.Cheng \& C.H.Yueh [23]. The four species of Gymnopetalum have been allocated to two sections that differ in flower morphology, the typical sect. Gymnopetalum with just one species from southern India and Sri Lanka and sect. Tripodanthera (M.Roem.) Cogn. with three southeast Asian and Malesian species [24].

Here we test the monophyly and phylogenetic placement of Trichosanthes using a broad sampling of some $60 \%$ of its species, including the type species of each section name, plus representatives of Gymnopetalum, Hodgsonia, and other Sicyoeae as well as more distant outgroups. The well-resolved phylogeny, combined with field observations on flower shape and color, allows us to test whether petal fringes in Old World Sicyoeae evolved just once as would be the case if Gymnopetalum and Hodgsonia were nested inside it [20] or multiple times as would be implied by these genera having separate evolutionary histories. A combination of molecular-dating and ancestral area reconstruction permits reconstructing the biogeographical history of the Trichosanthes clade.

\section{Results and discussion}

Phylogenetic analyses and taxonomy

Phylogenies obtained under Bayesian or Maximum Likelihood (ML) optimization revealed no statistically supported incongruences, defined as nodes with Bayesian posterior probabilities (PP) $>0.95$ or ML bootstrap support $>75$. A Bayesian consensus tree is shown in Figure 2. It reveals that the genus Trichosanthes is paraphyletic because Gymnopetalum is embedded in it, while Gymnopetalum is polyphyletic because its four species do not group together. Instead, G. tubiflorum (Wight \& Arn.) Cogn. groups with species from sections Trichosanthes and Cucumeroides (1.00 PP/84 ML support), while G. orientale W.J.de Wilde \& Duyfjes, G. chinense (Lour.) Merr., and G. scabrum (Lour.) W.J. de Wilde \& Duyfjes are sister to section Edulis (1.00 PP/86 ML). The Trichosanthes/Gymnopetalum clade (56 species sampled; 0.99 PP/62 ML support) is sister to Luffa, a genus of seven or eight species of which we included five. This sister group relationship, however, is only weakly supported (Figure 2). The genus Hodgsonia (two species with long-fringed flowers, 


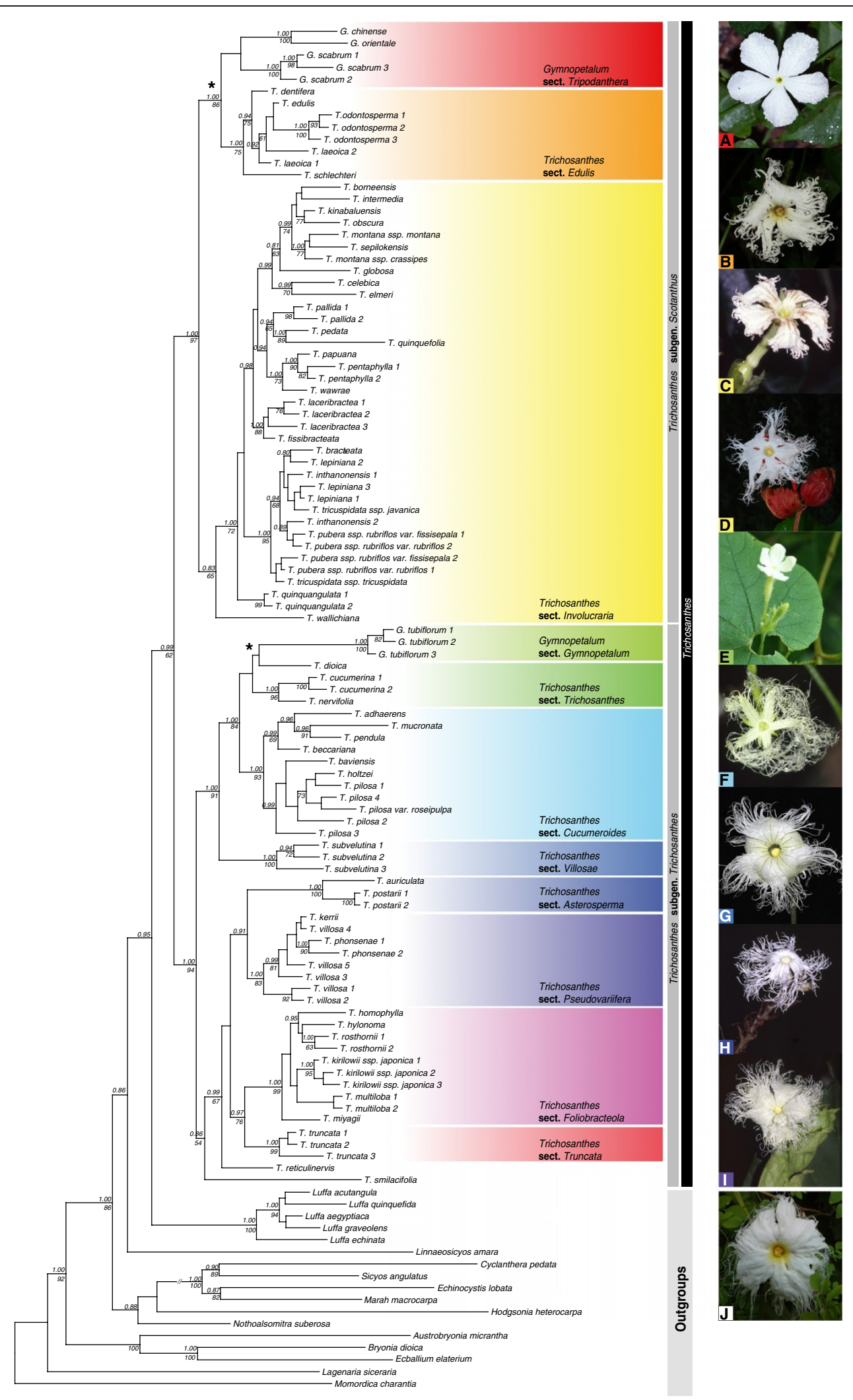

Figure 2 (See legend on next page.) 
(See figure on previous page.)

Figure 2 Bayesian consensus tree with posterior probabilities $(>0.80)$ and maximum likelihood bootstrap values $(>60 \%)$ shown at the nodes. Photos on the right illustrate the floral morphology of the different sections and belong to the following species: A) Gymnopetalum chinense; B) Trichosanthes odontosperma; C) Trichosanthes montana ssp. crassipes; D) Trichosanthes pubera ssp. rubriflos; E) Gymnopetalum tubiflorum; F) Trichosanthes beccariana; G) Trichosanthes subvelutina; H) Trichosanthes postarii; I) Trichosanthes villosa. Pictures courtesy of W. J. de Wilde and B. Duyfjes (A, C, D, F, H, I), W. E. Cooper (B), N. Filipowicz (E), H. Nicholson (G), and P. Brownless (J). Inferred losses of petal fringes are marked by an asterisk.

one sampled here) is only distantly related to the Trichosanthes/Gymnopetalum clade.

Of the seven sections previously proposed in Trichosanthes (see Background), three are supported by the molecular results, namely sections Asterosperma (1.00 $\mathrm{PP} / 100 \mathrm{ML}$; three species, two of them sampled here), Cucumeroides (1.00 PP/93 ML; seven species, five sampled), and Edulis (1.00 PP/75 ML; nine species, five sampled). Three other sections with more than one species (Involucraria, Foliobracteola, Trichosanthes) are not monophyletic in their current circumscriptions. To achieve a more natural classification, a revised infrageneric classification has been proposed including two new sections [17].

\section{The biogeographic history of the Trichosanthes clade}

Based on a fossil-calibrated Bayesian relaxed molecular clock model, Trichosanthes originated during the Oligocene (Figure 3), an estimate influenced by our prior constraint of the crown node of the Trichosanthes/ Gymnopetalum clade to $34 \mathrm{Ma}$. This constraint is based on Trichosanthes-like seeds from the Upper Eocene of Bulgaria [25] dating to c. $34 \mathrm{Ma}$ and seeds from the Oligocene of West Siberia [26] dating to c. 23.8 Ma [27]. Seeds assigned to Trichosanthes have also been reported from Miocene and Pliocene sites in France, Germany, Italy, and Poland [28-30], and Pliocene Trichosantheslike leaves are known from France [31]. The biogeographic analysis (Figure 4) inferred an East Asian origin of the genus (region $\mathrm{C}$ in Figure 4), but this inference is based only on the living species, while the just-discussed fossils indicate a more northern (Eurasian) range of Trichosanthes before the global climate cooling at the end of the Oligocene. Many other extinct elements of the European Oligocene, Miocene, and Pliocene floras, such as Taxodium, Craigia, Fagus kraeuselii, Ilex, and tropical Araceae, such as Caladiosoma, also have nearest living relatives in tropical Southeast Asia [31,32].

Collision between the Eurasian and Australian tectonic plates started in the Late Oligocene, about $25 \mathrm{Ma}$ ago, and the Sahul Shelf (carrying New Guinea) and Sunda Shelf (Sumatra, Java, and Borneo) reached their present proximity only by the Late Miocene, some $10 \mathrm{Ma}$ $[33,34]$. Mid-Miocene pollen records indicate a warm, moist climate and rainforest expansion on these newly forming islands [35], allowing groups adapted to humid forest conditions, such as the liana clade Trichosanthes, to spread and diversify. Such plant groups would have benefited from land bridges that during times of sea level changes repeatedly connected New Guinea and Australia on the one hand, and Indochina, Sumatra, Java, and Borneo on the other. The lowest sea levels, during the last glacial maximum (LGM), were approximately $120 \mathrm{~m}$ below those of today, resulting in the complete exposure of the Sunda Shelf; even sea level reduction by just $40 \mathrm{~m}$ already connected Indochina, Sumatra, Java, and Borneo [35,36]. No land bridges, however, ever connected the islands on the Sunda Shelf with those in "Wallacea," that is, Sulawesi, the Moluccas, and the Lesser Sunda Islands, or the latter with New Guinea and Australia on the Sahul Shelf. In zoogeography, these two boundaries are known as Wallace's Line and Lydekker's line, but their significance as floristic boundaries is doubtful $[37,38]$.

The most striking transoceanic disjunctions in Trichosanthes are numbered in Figure 4. They are (i) the disjunction between the Australian species T. subvelutina F.Muell. ex Cogn. and its sister clade on the Asian mainland and areas of the Sunda Shelf, dated to 23.8 (29.4-18.4) Ma; (ii) the disjunction between T. edulis Rugayah, T. dentifera Rugayah, T. laeoica C.Y.Cheng \& L.Q.Huang, T. schlechteri Harms from New Guinea, and T. odontosperma W.E.Cooper \& A.J.Ford from Australia on the one hand, and Gymnopetalum chinense, widespread in Asia as far East as Flores, and G. orientale in Sulawesi, the Lesser Sunda Islands, and the Moluccas on the other (this is dated to 16.7 (22.1-11.2) Ma, but the position of G. scabrum relative to G. chinense and $G$. orientale remains unclear; compare Figures 2, 3, and 4); and (iii) the disjunction between T. wawrae Cogn. from Thailand, peninsular Malaysia, Sumatra, and Borneo, and its sister clade T. papuana F.M.Bailey/T. pentaphylla F. Muell. ex Benth. from New Guinea and Australia, which dates to 7.1 (11.2-3.3) Ma.

Trichosanthes range expansion between New Guinea and Australia occurred during the Pliocene/Pleistocene, when these two regions were repeatedly connected due to the above-mentioned sea level changes [36]. Thus, the estimated divergence time of the Australian species T. odontosperma (a member of clade ii in Figure 4) from its New Guinean sister species, T. edulis, is 3.9 (6.4-1.6) Ma, while that of the sister species pair T. papuana from 


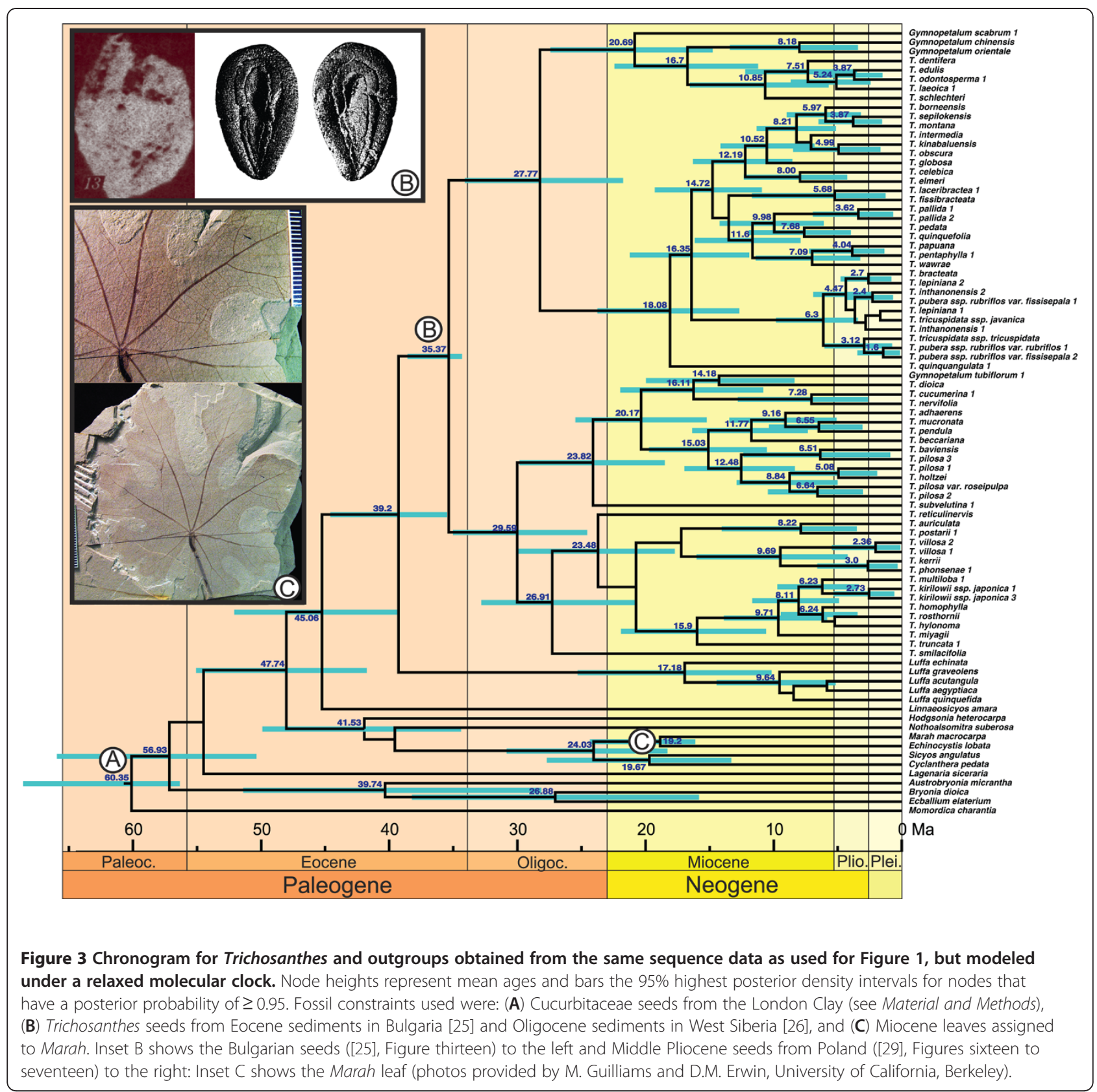

the Aru Islands and New Guinea, and T. pentaphylla from Australia (clade iii in Figure 4) is 4.0 (7.1-1.4) Ma; considering their error ranges, these ages fall in the Pliocene/Pleistocene.

The geographic history of $T$. pilosa Lour. (including the synonyms T. baviensis Gagnep. and T. holtzei F.Muell. [16]), a widespread species here represented by seven samples from Queensland (Australia), Thailand, Vietnam, and Japan, cannot be inferred because the within-species relationships lack statistical support (Figure 2). Inferring the origin of the snake gourd, T. cucumerina, a vegetable cultivated in tropical and subtropical regions around the globe (represented by a single sample from Sri Lanka) also would require population-level sampling. Both species have fleshy red fruits and small seeds, probably dispersed by birds.

\section{Evolution and loss of petal fringes}

The phylogeny obtained here implies that long-fringed corollas evolved independently in the Asian genera Hodgsonia and Trichosanthes and were lost in three of the four species formerly placed in the genus Gymnopetalum (petals still bear c. $5 \mathrm{~mm}$-long fringes in G. orientale). The two inferred losses (marked with an asterisk 


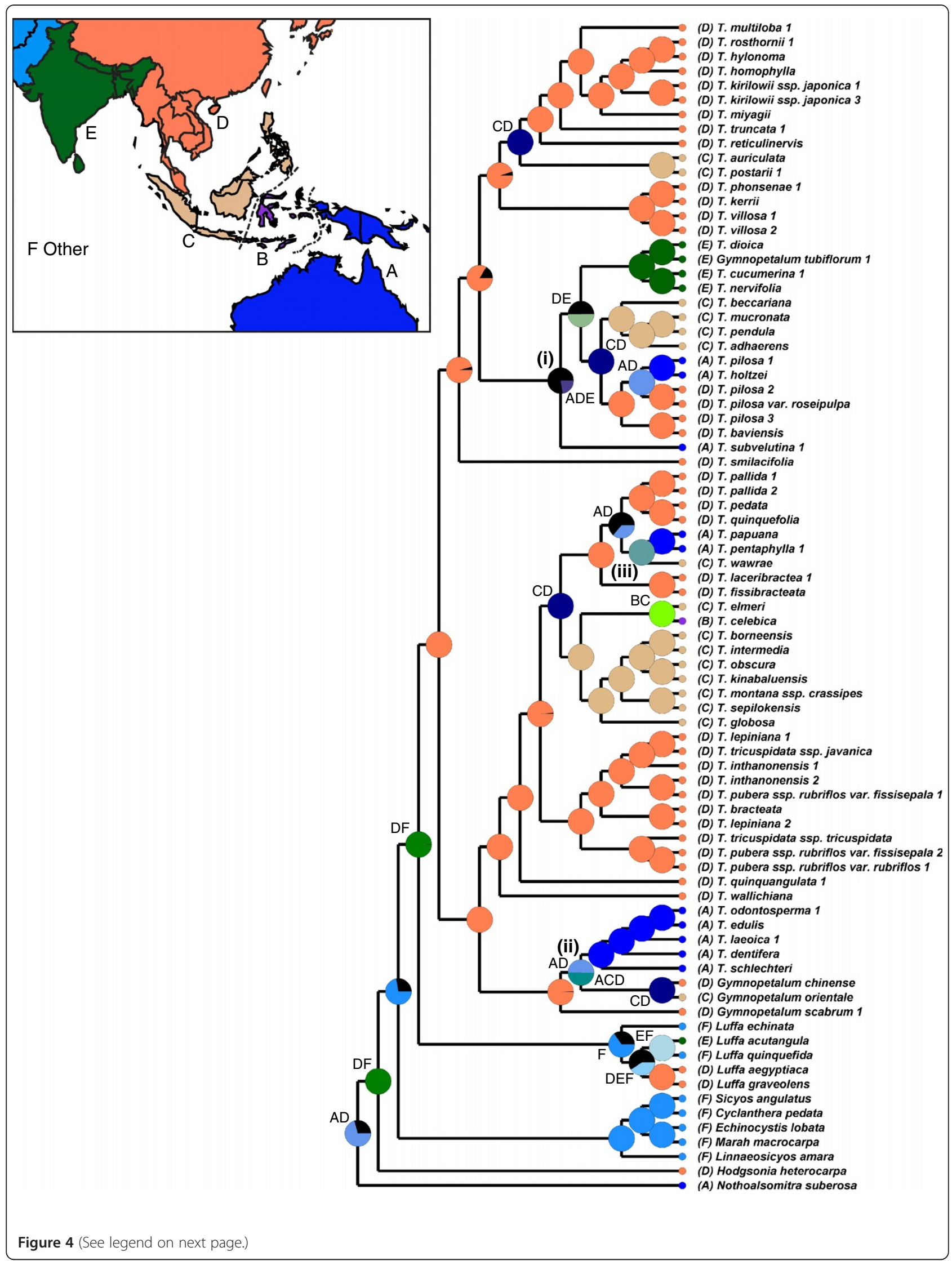


in Figure 2) coincide with shifts from nocturnal to diurnal flowering times (HS personal observation of G. scabrum and G. chinense in Cambodia, Jan. 2010, and China, Sept. 2005; N. Filipowicz, Medical University of Gdansk, personal observation of G. tubiflorum in India, Nov. 2010), and it therefore seems likely that there is a shift from predominantly nocturnal sphingid pollinators to diurnal bee or butterfly pollinators. The loss of fringes does not coincide with long-distance dispersal events to insular habitats (where hawkmoths might be absent), and the trigger for the pollinator shifts so far is unknown.

The adaptive function of the corolla fringes in pollinator attraction requires experimental study. An innate preference for radial patterns [39] and high contrasts might help hawkmoths find their nectar sources $[5,6]$, and one possible explanation for the evolution of fringed petals is that they help create such a radial pattern and sharper contrasts between the petals and a dark background [4]. In a diurnal, hawkmoth-pollinated Viola species, more complex corolla outlines correlate with higher fruit set [40] but it remains to be tested if this is also the case in the nocturnal Trichosantheshawkmoth system. Another untested possibility is that the fringes with their highly increased surface area and exposed position might be involved in scent production (B. Schlumpberger, Herrenhaeuser Gardens, Hannover, pers. comm., Feb. 2012) or produce a waving motion, which has been shown to increase pollinator attraction in other systems [41]. Anatomical studies of the petal tissue of Trichosanthes, wind tunnel experiments with naive hawkmoths, and detailed field observations are required to test these possibilities.

\section{Conclusions}

Molecular evidence supports the inclusion of Gymnopetalum into a then monophyletic Trichosanthes [17]. Our molecular phylogenies reveal that long-fringed petals evolved independently in Hodgsonia and Trichosanthes/ Gymnopetalum, followed by two losses of corolla fringes in the latter clade, most likely associated with pollinator shifts. Molecular dating and a biogeographic analysis indicate an Oligocene initial diversification of Trichosanthes in mainland Asia. The lineage then diversified and spread in Malaysia (the Malesian biogeographic region) during the late Miocene and Pliocene, reaching the Australian continent several times.

\section{Methods}

\section{Morphology}

Herbarium specimens from A, BRI, CNS, E, GH, K, KUN, KYO, L, LE, M, MO, P, S, UC, UPS and US were obtained on loan or studied during herbarium visits. Determination of herbarium material was verified using identification keys [9,11-16,19,42]. All species in Trichosanthes have corolla fringes, and these are absent in three of the four Gymnopetalum species, except G. orientale, which can have short-fimbriate petal margins (fringes up to $5 \mathrm{~mm}$ length).

\section{Sampling, DNA extraction and amplification}

We included six DNA regions, namely the nuclear ribosomal ITS region (ITS1-5.8S-ITS2), the chloroplast genes $r b c L$ and $m a t K$, the $\operatorname{trn} L$ and $\operatorname{trn} L$-trnF intron and spacer, and rpl20-rps12 spacer. Data for $r b c L$ and the trnL region were taken from previous studies $[7,18,20,43,44]$. Only plant samples for which two or more markers were successfully sequenced were included in the analyses, and the combined dataset included one of the two species of Hodgsonia, all four of Gymnopetalum, and 52 of Trichosanthes, representing approximately $60 \%$ of the accepted species in the latter genus. Type species of all sections were included: Gymnopetalum tubiflorum (Wight \& Arn.) Cogn. (G. sect. Gymnopetalum), Gymnopetalum chinense (Lour.) Merr. (G. sect. Tripodanthera), Trichosanthes postarii W.J.de Wilde \& Duyfjes (T. sect. Asterosperma), Trichosanthes pilosa Lour. (T. sect. Cucumeroides), Trichosanthes edulis Rugayah (T. sect. Edulis), Trichosanthes kirilowii Maxim. (T. sect. Foliobracteola), Trichosanthes wallichiana (Ser.) Wight (T. sect. Involucraria), Trichosanthes villosa Blume (T. sect. Pseudovariifera), Trichosanthes cucumerina L. (T. sect. Trichosanthes), Trichosanthes truncata C.B. Clarke (T. sect. Truncata), Trichosanthes subvelutina F. Muell. ex Cogn. (T. sect. Villosae). Species names and their authors, specimen voucher information, and GenBank accession numbers for all sequenced markers (including 262 new sequences) are summarized in Table 1.

Total DNA was extracted using the Carlson/Yoon DNA isolation procedure [45] and a Mini-Beadbeater (BioSpec Products) to pulverize the plant material. Extracts were purified using the GE Illustra GFX ${ }^{\mathrm{mm}}$ PCR DNA and Gel Band Purification Kit following the standard protocol. 
Table 1 Voucher information and GenBank accession numbers

\begin{tabular}{|c|c|c|c|c|c|c|c|c|c|}
\hline Species & No. & Voucher (Herbarium) & Origin of the sequenced material & ITS & rp/20-rps12 IS & matK & $r b c L$ & trnL-trnF IS & trnL intron \\
\hline $\begin{array}{l}\text { Austrobryonia micrantha } \\
\text { (F.Muell.) I.Telford }\end{array}$ & & I. R. Telford 8173 (CANB) & Australia, New South Wales & EF487546 & EF487567 & EF487559 & EF487552 & EF487575 & EF487575 \\
\hline \multirow[t]{2}{*}{ Bryonia dioica Jacq. } & & (1) S. Renner 2187 (M) & (1) Switzerland, cult. BG Zürich & (2) EU102709 & (1) DQ648157 & (1) DQ536641 & (1) DQ536791 & (1) DQ536791 & (1) DQ536791 \\
\hline & & (2) A. Faure 66/76 (M) & (2) Algeria, Lamoriciere & & & & & & \\
\hline $\begin{array}{l}\text { Cyclanthera pedata } \\
\text { (L.) Schrad. }\end{array}$ & & S. Renner et al. 2767 (M) & Germany, cult. BG Mainz & HE661293 & DQ648172 & DQ536667 & DQ535749 & DQ536767 & DQ536767 \\
\hline \multirow{2}{*}{$\begin{array}{l}\text { Ecballium elaterium (L.) } \\
\text { A.Rich. ssp. elaterium }\end{array}$} & & (1) M. Chase $922(\mathrm{~K})$ & (1) UK, cult. RBG-K & (2) EU102746 & (1) AY968541 & (1) AY973019 & (1) AY973023 & (1) AY973006 & (1) AY973006 \\
\hline & & (2) S. Renner et al. 2768 (M) & (2) Germany, cult. BG Mainz & & & & & & \\
\hline $\begin{array}{l}\text { Echinocystis lobata } \\
\text { (Michx.) Torr. \& A.Gray }\end{array}$ & & S. Renner et al. 2829 (M) & Germany, cult. BG Mainz & - & DQ648174 & DQ536673 & DQ535809 & DQ536814 & DQ536814 \\
\hline $\begin{array}{l}\text { Gymnopetalum chinense } \\
\text { (Lour.) Merr. }\end{array}$ & & H. Schaefer 2005/661 (M) & China, Guangdong & HE661294 & EU155612 & EU155606 & EU155601 & EU155621 & EU155630 \\
\hline $\begin{array}{l}\text { Gymnopetalum orientale } \\
\text { W.J. de Wilde \& Duyfjes }\end{array}$ & & M. van Balgooy 7553 (L) & Indonesia, Bali & HE661301 & HE661468 & HE661397 & - & - & - \\
\hline $\begin{array}{l}\text { Gymnopetalum scabrum } \\
\text { (Lour.) W.J. de Wilde \& } \\
\text { Duyfjes }\end{array}$ & 1 & $\begin{array}{l}\text { W. de Wilde \& B. Duyfjes } \\
22269(\mathrm{~L})\end{array}$ & Thailand, Central & HE661295 & DQ536556 & DQ536683 & DQ535754 & DQ536824 & DQ536824 \\
\hline $\begin{array}{l}\text { Gymnopetalum scabrum } \\
\text { (Lour.) W.J. de Wilde \& } \\
\text { Duyfjes }\end{array}$ & 2 & J. Maxwell 16-11-2002 (CMU) & Thailand & HE661296 & HE661469 & HE661398 & - & - & - \\
\hline $\begin{array}{l}\text { Gymnopetalum scabrum } \\
\text { (Lour.) W.J. de Wilde \& } \\
\text { Duyfjes }\end{array}$ & 3 & $\begin{array}{l}\text { C.H. Wong, J. Helm \& } \\
\text { J. Schultze-Motel } 2071 \text { (LE) }\end{array}$ & China, Hainan & HE661297 & HE661470 & HE661399 & - & - & - \\
\hline $\begin{array}{l}\text { Gymnopetalum tubiflorum } \\
\text { (Wight \& Arn.) Cogn. }\end{array}$ & 1 & $\begin{array}{l}\text { N. Filipowicz \& Z. Van } \\
\text { Herwijnen NF25a (M) }\end{array}$ & India, Kerala & HE661298 & HE661471 & HE661400 & - & - & - \\
\hline $\begin{array}{l}\text { Gymnopetalum tubiflorum } \\
\text { (Wight \& Arn.) Cogn. }\end{array}$ & 2 & A. Alston $1670(\mathrm{UC})$ & Sri Lanka, Veragantota & HE661299 & HE661472 & HE661401 & - & - & - \\
\hline $\begin{array}{l}\text { Gymnopetalum tubiflorum } \\
\text { (Wight \& Arn.) Cogn. }\end{array}$ & 3 & G.H.K. Thwaites CP1625 (K) & Sri Lanka & HE661300 & HE661473 & HE661402 & - & - & - \\
\hline \multirow{2}{*}{$\begin{array}{l}\text { Hodgsonia heteroclita } \\
\text { Hook.f. \& Thomson }\end{array}$} & & (1) P. Phonsena 4705 (L) & (1) Thailand, Nan & (1) HE661302 & (1) HE661474 & (1) HE661403 & - & (2) EU155631 & - \\
\hline & & (2) L. Loeffler s.n. (M) & (2) Bangladesh & & & & & & \\
\hline $\begin{array}{l}\text { Lagenaria siceraria (Molina) } \\
\text { Standl. }\end{array}$ & & M. Merello 1331 (MO) & Ghana & HE661303 & HE661475 & HE661404 & AY935747 & AY935788 & AY968570 \\
\hline $\begin{array}{l}\text { Linnaeosicyos amara (L.) } \\
\text { H.Schaef. \& Kocyan }\end{array}$ & & $\begin{array}{l}\text { M. Mejia, J. Pimentel \& } \\
\text { R. Garcia } 1877 \text { (NY) }\end{array}$ & Dominican Republic & HE661304 & DQ536602 & DQ536741 & DQ535774 & DQ536873 & DQ536873 \\
\hline \multirow[t]{2}{*}{ Luffa acutangula (L.) Roxb. } & & $\begin{array}{l}\text { (1) S. Renner et al. } 2757 \text { (M), } \\
\text { seeds from D. S. Decker-Walters } \\
\text { \& A. Wagner TCN } 1130 \text { (FTG) }\end{array}$ & $\begin{array}{l}\text { (1) Germany, cult. BG Munich, } \\
\text { seeds from India, Ahmadnagar, } \\
\text { Maharasthra }\end{array}$ & (1) HE661305 & (1) HE661476 & (2) DQ536695 & (2) DQ535826 & (2) DQ536835 & (2) DQ536835 \\
\hline & & (2) L.X. Zhou s.n., no voucher & (2) China, cult. BG Guangzhou & & & & & & \\
\hline
\end{tabular}


Table 1 Voucher information and GenBank accession numbers (Continued)

\begin{tabular}{|c|c|c|c|c|c|c|c|c|c|}
\hline $\begin{array}{l}\text { Luffa aegyptiaca Mill. } \\
\text { (incl. L. cylindrica L.) }\end{array}$ & & $\begin{array}{l}\text { D.Z. Zhang } 15 \text { April 2003, } \\
\text { no voucher }\end{array}$ & China, cult. BG Guangzhou & HE661306 & HE661477 & HE661405 & DQ535827 & DQ536836 & DQ536836 \\
\hline Luffa echinata Roxb. & & G. Schweinfurth 555 (M) & Egypt & HE661307 & HE661478 & HE661406 & - & EU436357 & EU436357 \\
\hline Luffa graveolens Roxb. & & $\begin{array}{l}\text { S. Renner \& A. Kocyan } 2758 \text { (M), } \\
\text { seeds from D. Decker-Walters } \\
1543 \text { (FTG 121855) }\end{array}$ & $\begin{array}{l}\text { Germany, cult. BG Munich, } \\
\text { seeds from India, USDA PI540921 }\end{array}$ & HE661308 & EU436334 & EU436409 & EU436385 & EU436358 & EU436358 \\
\hline \multirow{2}{*}{\multicolumn{2}{|c|}{$\begin{array}{l}\text { Luffa quinquefida } \\
\text { (Hook. \& Arn.) Seemann }\end{array}$}} & (1) R. Berhaut 7308 (M) & (1) Senegal & (2) HQ201986 & (1) EU436335 & (2) DQ536697 & - & (1) EU436359 & - \\
\hline & & $\begin{array}{l}\text { (2) S. Renner \& A. Kocyan } \\
2754 \text { (M), seeds from D. S. } \\
\text { Decker-Walters TCN } 1440 \\
\text { (FTG 118010) }\end{array}$ & $\begin{array}{l}\text { (2) Germany, cult. BG Munich, } \\
\text { seeds originally from Louisiana, } \\
\text { USA }\end{array}$ & & & & & & \\
\hline \multirow{2}{*}{\multicolumn{2}{|c|}{$\begin{array}{l}\text { Marah macrocarpa } \\
\text { (Greene) Greene }\end{array}$}} & (1) M. Olson s.n. (MO) & (1) USA, Sonoran Desert & (2) AF11906-7 & (1) DQ536566 & (2) AY968453 & (2) AY968524 & (1) AY968387 & (1) AY968571 \\
\hline & & $\begin{array}{l}\text { (2) D. Arisa \& S. Swensen } \\
1009 \text { (RSA) }\end{array}$ & (2) USA, Sonoran Desert & & & & & & \\
\hline \multicolumn{2}{|l|}{ Momordica charantia L. } & S. Renner et al. 2775 (M) & Germany, cult. BG Munich & HE661309 & DQ491013 & DQ491019 & DQ535760 & DQ501269 & DQ501269 \\
\hline \multicolumn{2}{|l|}{$\begin{array}{l}\text { Nothoalsomitra suberosa } \\
\text { (F.M.Bailey) I.Telford }\end{array}$} & I. Telford 12487 (NE) & Australia, SE Queensland & HE661310 & DQ536575 & DQ536709 & DQ535762 & DQ536844 & DQ536844 \\
\hline \multicolumn{2}{|l|}{ Sicyos angulatus $\mathrm{L}$. } & M. Chase $979(K)$ & North America & HE661311 & DQ648189 & DQ536732 & DQ535847 & DQ536777 & DQ536777 \\
\hline \multicolumn{2}{|l|}{$\begin{array}{l}\text { Trichosanthes adhaerens } \\
\text { W.J. de Wilde \& Duyfjes }\end{array}$} & $\begin{array}{l}\text { S. Lim, J. J. Postar \& G. Markus } \\
\text { SAN } 143273 \text { (L) }\end{array}$ & Malaysia, Borneo, Sabah & HE661312 & HE661479 & - & - & - & - \\
\hline \multicolumn{2}{|l|}{$\begin{array}{l}\text { Trichosanthes auriculata } \\
\text { Rugayah }\end{array}$} & $\begin{array}{l}\text { A. Kalat, I. Abdullah, \& J. Clayton } \\
\text { BRUN } 17016 \text { (L) }\end{array}$ & Borneo, Brunei & HE661313 & HE661480 & HE661407 & - & - & - \\
\hline \multicolumn{2}{|l|}{$\begin{array}{l}\text { Trichosanthes baviensis } \\
\text { Gagnep. }\end{array}$} & N.M. Cuong 1248 (P) & Vietnam & HE661314 & HE661481 & - & - & - & - \\
\hline \multicolumn{2}{|l|}{$\begin{array}{l}\text { Trichosanthes beccariana } \\
\text { Cogn. ssp. beccariana }\end{array}$} & W. de Wilde et al. SAN 142229 (L) & Malaysia, Borneo, Sabah & HE661315 & HE661482 & HE661408 & - & - & - \\
\hline \multicolumn{2}{|l|}{$\begin{array}{l}\text { Trichosanthes borneensis } \\
\text { Cogn. }\end{array}$} & C. Argent et al. 93127 (E) & $\begin{array}{l}\text { Indonesia, Borneo, Kalimantan } \\
\text { Timur }\end{array}$ & HE661316 & HE661483 & - & - & - & - \\
\hline \multicolumn{2}{|l|}{$\begin{array}{l}\text { Trichosanthes bracteata } \\
\text { (Lam.) Voigt }\end{array}$} & T. Haegele $20(\mathrm{M})$ & India, Kochin & HE661317 & HE661484 & EU155608 & EU155602 & EU155622 & EU155632 \\
\hline \multicolumn{2}{|l|}{$\begin{array}{l}\text { Trichosanthes celebica } \\
\text { Cogn. }\end{array}$} & W. de Wilde \& B. Duyfjes $21903(\mathrm{~L})$ & Indonesia, Sulawesi & HE661318 & HE661485 & HE661409 & - & - & - \\
\hline Trichosanthes cucumerina $\mathrm{L}$. & 1 & H. Schaefer 2007/327 (M) & Germany, cult. BG Munich & HE661319 & EU155614 & EU155609 & EU155603 & EU155623 & EU155633 \\
\hline Trichosanthes cucumerina $\mathrm{L}$. & 2 & N. Lundqvist 11380 (UPS) & Sri Lanka & HE661320 & HE661486 & HE661410 & - & - & - \\
\hline \multicolumn{2}{|l|}{$\begin{array}{l}\text { Trichosanthes dentifera } \\
\text { Rugayah }\end{array}$} & J.H.L. Waterhouse 445-B (L) & $\begin{array}{l}\text { Papua New Guinea, } \\
\text { Bougainville Is. }\end{array}$ & HE661321 & HE661487 & - & - & - & - \\
\hline \multicolumn{2}{|l|}{ Trichosanthes dioica Roxb. } & $\begin{array}{l}\text { O. Polunin, W. Sykes \& J. Williams } \\
5925 \text { (E) }\end{array}$ & Nepal & HE661322 & HE661488 & HE661411 & - & - & - \\
\hline \multicolumn{2}{|l|}{$\begin{array}{l}\text { Trichosanthes edulis } \\
\text { Rugayah }\end{array}$} & W. Avé 4076 (L) & Indonesia, Irian Jaya & HE661323 & HE661489 & HE661412 & - & - & - \\
\hline
\end{tabular}


Table 1 Voucher information and GenBank accession numbers (Continued)

\begin{tabular}{|c|c|c|c|c|c|c|c|c|c|}
\hline Trichosanthes elmeri Merr. & & E.F.J. Campbell 43 (E) & Malaysia, Borneo, Sabah & HE661324 & HE661490 & - & - & - & - \\
\hline Trichosanthes globosa Blume & & W. de Wilde et al. SAN 144003 (L) & Malaysia, Borneo, Sabah & HE661325 & HE661491 & HE661413 & - & - & - \\
\hline Trichosanthes holtzei F.Muell. & & B. Gray 7482 (CNS) & Australia, N Queensland & HE661326 & HE661492 & HE661414 & - & - & - \\
\hline $\begin{array}{l}\text { Trichosanthes homophylla } \\
\text { Hayata }\end{array}$ & & Y.-C. Kao $499(\mathrm{GH})$ & Taiwan & HE661327 & HE661493 & HE661415 & - & - & - \\
\hline $\begin{array}{l}\text { Trichosanthes hylonoma } \\
\text { Hand.-Mazz. }\end{array}$ & & Wuling Mt Exp 1646 (KUN) & China & HE661328 & HE661494 & HE661416 & - & - & - \\
\hline $\begin{array}{l}\text { Trichosanthes intermedia } \\
\text { W.J. de Wilde \& Duyfjes }\end{array}$ & & V. Julaihi et al. S 76602 (L) & Malaysia, Borneo, Sarawak & HE661329 & HE661495 & - & - & - & - \\
\hline $\begin{array}{l}\text { Trichosanthes inthanonensis } \\
\text { Duyfjes \& Pruesapan }\end{array}$ & 1 & $\begin{array}{l}\text { P. Phonsena, W. de Wilde \& } \\
\text { B. Duyfjes } 3930(\mathrm{~L})\end{array}$ & Thailand, Chiang Mai & HE661330 & HE661496 & HE661417 & - & - & - \\
\hline $\begin{array}{l}\text { Trichosanthes inthanonensis } \\
\text { Duyfjes \& Pruesapan }\end{array}$ & 2 & K. Pruesapan et al. 67 (L) & Thailand, Kanchanaburi & HE661331 & HE661497 & HE661418 & - & - & - \\
\hline Trichosanthes kerrii Craib & & $\begin{array}{l}\text { P. Phonsena, W. de Wilde \& } \\
\text { B. Duyfjes } 3959(\mathrm{~L})\end{array}$ & Thailand, Nan & HE661333 & HE661498 & - & - & - & - \\
\hline $\begin{array}{l}\text { Trichosanthes kinabaluensis } \\
\text { Rugayah }\end{array}$ & & J. Postar et al. SAN 144260 (L) & Malaysia, Borneo, Sabah & HE661334 & EU155615 & HE661419 & - & EU155624 & EU155634 \\
\hline $\begin{array}{l}\text { Trichosanthes kirilowii Maxim. } \\
\text { var. japonica (Miq.) Kitam. }\end{array}$ & 3 & H. Takahashi 20711 (GIFU) & Japan & HE661335 & DQ536603 & DQ536742 & DQ535855 & DQ536874 & DQ536874 \\
\hline $\begin{array}{l}\text { Trichosanthes kirilowii Maxim. } \\
\text { var. japonica (Miq.) Kitam. }\end{array}$ & 1 & K. Kondo 05090401e (KYO) & Japan & HE661332 & HE661499 & HE661420 & - & - & - \\
\hline $\begin{array}{l}\text { Trichosanthes kirilowii Maxim. } \\
\text { var. japonica (Miq.) Kitam. }\end{array}$ & 2 & $\begin{array}{l}\text { K. Deguchi, K. Uchida, } \\
\text { K. Shiino \& H. Hideshima } \\
\text { s.n. (KYO) }\end{array}$ & Japan & - & HE661500 & HE661421 & - & - & - \\
\hline $\begin{array}{l}\text { Trichosanthes laceribractea } \\
\text { Hayata }\end{array}$ & 1 & S. Fujii 9623 (KYO) & Japan & HE661336 & HE661501 & HE661422 & - & - & - \\
\hline $\begin{array}{l}\text { Trichosanthes laceribractea } \\
\text { Hayata }\end{array}$ & 2 & S. Fujii 9978 (KYO) & Japan & HE661337 & HE661502 & HE661423 & - & - & - \\
\hline $\begin{array}{l}\text { Trichosanthes laceribractea } \\
\text { Hayata }\end{array}$ & 3 & Liang Deng 7090 (KUN) & China & HE661338 & HE661503 & - & - & - & - \\
\hline $\begin{array}{l}\text { Trichosanthes laeoica } \\
\text { C.Y.Cheng \& L.Q.Huang }\end{array}$ & 1 & M. Coode et al. NGF 32585 (E) & $\begin{array}{l}\text { Papua New Guinea, } \\
\text { Eastern Highlands }\end{array}$ & HE661339 & HE661504 & - & - & - & - \\
\hline $\begin{array}{l}\text { Trichosanthes laeoica } \\
\text { C.Y.Cheng \& L.Q.Huang }\end{array}$ & 2 & P. Katik LAE 77807a (BRI) & Papua New Guinea & HE661340 & HE661505 & - & - & - & - \\
\hline $\begin{array}{l}\text { Trichosanthes lepiniana } \\
\text { (Naud.) Cogn. }\end{array}$ & 1 & J.D.A. Stainton 8522 (E) & Nepal & HE661341 & HE661506 & HE661424 & - & - & - \\
\hline $\begin{array}{l}\text { Trichosanthes lepiniana } \\
\text { (Naud.) Cogn. }\end{array}$ & 2 & Shanzu Wen 85 (KUN) & China & HE661342 & HE661507 & HE661425 & - & - & - \\
\hline $\begin{array}{l}\text { Trichosanthes lepiniana } \\
\text { (Naud.) Cogn. }\end{array}$ & 3 & H. de Boer HB49, coll. 1865 (P) & France, cult BG Paris & HE661343 & HE661508 & - & - & - & - \\
\hline
\end{tabular}


Table 1 Voucher information and GenBank accession numbers (Continued)

\begin{tabular}{|c|c|c|c|c|c|c|c|c|c|}
\hline \multicolumn{2}{|l|}{$\begin{array}{l}\text { Trichosanthes miyagii } \\
\text { Hayata }\end{array}$} & T. Yamazaki $310(\mathrm{KYO})$ & Japan & HE661344 & HE661509 & HE661426 & - & - & - \\
\hline \multicolumn{2}{|l|}{$\begin{array}{l}\text { Trichosanthes montana } \\
\text { Rugayah ssp. crassipes W.J. de } \\
\text { Wilde \& Duyfjes }\end{array}$} & J. Postar et al. SAN 144259 (L) & Malaysia, Borneo, Sabah & HE661346 & EU155616 & HE661427 & - & EU155625 & EU155635 \\
\hline \multicolumn{2}{|l|}{$\begin{array}{l}\text { Trichosanthes montana } \\
\text { Rugayah ssp. montana }\end{array}$} & W. de Wilde et al. 22279 (L) & Indonesia, Java & HE661345 & HE661510 & - & - & - & - \\
\hline \multicolumn{2}{|l|}{$\begin{array}{l}\text { Trichosanthes mucronata } \\
\text { Rugayah }\end{array}$} & $\begin{array}{l}\text { W. de Wilde \& B. Duyfjes SAN } \\
139459 \text { (L) }\end{array}$ & Malaysia, Borneo, Sabah & HE661347 & HE661511 & HE661428 & - & - & - \\
\hline $\begin{array}{l}\text { Trichosanthes multiloba } \\
\text { Miq. }\end{array}$ & 1 & $\begin{array}{l}\text { S. Tsugaru, G. Murata \& } \\
\text { T. Sawada s.n. (KYO) }\end{array}$ & Japan & HE661348 & HE661512 & HE661429 & - & - & - \\
\hline $\begin{array}{l}\text { Trichosanthes multiloba } \\
\text { Miq. }\end{array}$ & 2 & S. Fujii 9957 (KYO) & Japan & HE661349 & HE661513 & HE661430 & - & - & - \\
\hline \multicolumn{2}{|l|}{ Trichosanthes nervifolia $\mathrm{L}$. } & B. Jonsell 3828 (UPS) & Sri Lanka & HE661350 & HE661514 & HE661431 & - & - & - \\
\hline \multicolumn{2}{|l|}{$\begin{array}{l}\text { Trichosanthes obscura } \\
\text { Rugayah }\end{array}$} & K.M. Wang 1581 (L) & Borneo, Brunei & HE661351 & HE661515 & - & - & - & - \\
\hline $\begin{array}{l}\text { Trichosanthes odontosperma } \\
\text { W.E.Cooper \& A.J.Ford }\end{array}$ & 1 & H. Schaefer 2007/09 (M) & Australia, Queensland & HE661352 & EU037013 & HE661432 & - & EU037011 & EU037010 \\
\hline $\begin{array}{l}\text { Trichosanthes odontosperma } \\
\text { W.E.Cooper \& A.J.Ford }\end{array}$ & 2 & B. Gray 9147 (UPS) & Australia, Queensland & HE661353 & HE661516 & HE661433 & - & - & - \\
\hline $\begin{array}{l}\text { Trichosanthes odontosperma } \\
\text { W.E.Cooper \& A.J.Ford }\end{array}$ & 3 & 1. Telford 11285 (CNS) & Australia, Queensland & HE661354 & HE661517 & HE661434 & - & - & - \\
\hline $\begin{array}{l}\text { Trichosanthes pallida } \\
\text { Duyfjes \& Pruesapan }\end{array}$ & 1 & $\begin{array}{l}\text { P. Phonsena, W. de Wilde \& } \\
\text { B. Duyfjes } 4658(\mathrm{~L})\end{array}$ & Thailand, Phetchaburi & HE661355 & HE661518 & HE661435 & - & - & - \\
\hline $\begin{array}{l}\text { Trichosanthes pallida } \\
\text { Duyfjes \& Pruesapan }\end{array}$ & 2 & $\begin{array}{l}\text { P. Phonsena, W. de Wilde \& } \\
\text { B. Duyfjes } 3981 \text { (L) }\end{array}$ & Thailand, Phetchaburi & HE661356 & HE661519 & HE661436 & - & - & - \\
\hline $\begin{array}{l}\text { Trichosanthes papuana } \\
\text { F.M.Bailey }\end{array}$ & & $\begin{array}{l}\text { W. Takeuchi \& D. Ama } \\
17069(\mathrm{~L})\end{array}$ & Papua New Guinea & HE661357 & HE661520 & HE661437 & - & - & - \\
\hline $\begin{array}{l}\text { Trichosanthes pedata } \\
\text { Merr. \& Chun }\end{array}$ & & Jiangiang Li 239 (KUN) & China & HE661358 & HE661521 & HE661438 & - & - & - \\
\hline $\begin{array}{l}\text { Trichosanthes pendula } \\
\text { Rugayah }\end{array}$ & & J. Postar et al. 144100 (L) & Malaysia, Borneo, Sabah & HE661359 & EU155617 & HE661439 & - & EU155626 & EU155636 \\
\hline $\begin{array}{l}\text { Trichosanthes pentaphylla } \\
\text { F.Muell. ex Benth. }\end{array}$ & 1 & W. Cooper 2094 (CNS) & Australia, Queensland & HE661360 & HE661522 & HE661440 & - & - & - \\
\hline $\begin{array}{l}\text { Trichosanthes pentaphylla } \\
\text { F.Muell. ex Benth. }\end{array}$ & 2 & W. Cooper 2061 (CNS) & Australia, Queensland & HE661361 & HE661523 & HE661441 & - & - & - \\
\hline $\begin{array}{l}\text { Trichosanthes phonsenae } \\
\text { Duyfjes \& Pruesapan }\end{array}$ & 1 & $\begin{array}{l}\text { P. Phonsena, W. de Wilde \& } \\
\text { B. Duyfjes } 4419(\mathrm{~L})\end{array}$ & Thailand, Phetchaburi & HE661362 & HE661524 & HE661442 & - & - & - \\
\hline $\begin{array}{l}\text { Trichosanthes phonsenae } \\
\text { Duyfjes \& Pruesapan }\end{array}$ & 2 & $\begin{array}{l}\text { P. Phonsena, W. de Wilde \& } \\
\text { B. Duyfjes } 3980(\mathrm{~L})\end{array}$ & Thailand, Phetchaburi & HE661363 & HE661525 & HE661443 & - & - & - \\
\hline
\end{tabular}


Table 1 Voucher information and GenBank accession numbers (Continued)

\begin{tabular}{|c|c|c|c|c|c|c|c|c|c|}
\hline Trichosanthes pilosa Lour. & 1 & H. Schaefer 2007/17 (M) & Australia, Queensland & HE661364 & EU155620 & EU155611 & - & EU155629 & EU155639 \\
\hline Trichosanthes pilosa Lour. & 2 & $\begin{array}{l}\text { P. Phonsena, W. de Wilde \& } \\
\text { B. Duyfjes } 3913(\mathrm{~L})\end{array}$ & Thailand, Chiang Mai & HE661365 & HE661526 & HE661444 & - & - & - \\
\hline Trichosanthes pilosa Lour. & 3 & H. Takahashi 20755 (GIFU) & Japan & - & DQ536604 & DQ536743 & DQ535856 & DQ536875 & DQ536875 \\
\hline Trichosanthes pilosa Lour. & 4 & H. Schaefer 2007/09 (M) & Australia, Queensland & HE661366 & HE661528 & HE661445 & - & - & - \\
\hline $\begin{array}{l}\text { Trichosanthes pilosa var. } \\
\text { roseipulpa W.J. de Wilde \& } \\
\text { Duyfjes }\end{array}$ & & $\begin{array}{l}\text { P. Phonsena, W. de Wilde \& } \\
\text { B. Duyfjes } 4694 \text { (L, holotype) }\end{array}$ & Thailand, Nan & HE661367 & HE661529 & HE661446 & - & - & - \\
\hline $\begin{array}{l}\text { Trichosanthes postarii } \\
\text { W.J. de Wilde \& Duyfjes }\end{array}$ & 1 & $\begin{array}{l}\text { J. Postar et al. SAN } 144066 \\
\text { (L, isotype) }\end{array}$ & Malaysia, Borneo, Sabah & HE661368 & EU155618 & HE661447 & - & EU155627 & EU155637 \\
\hline $\begin{array}{l}\text { Trichosanthes postarii } \\
\text { W.J. de Wilde \& Duyfjes }\end{array}$ & 2 & J. Postar et al. SAN 144098 (L) & Malaysia, Borneo, Sabah & HE661369 & HE661530 & HE661448 & - & - & - \\
\hline $\begin{array}{l}\text { Trichosanthes pubera Blume } \\
\text { ssp. rubriflos (Cayla) Duyfjes \& } \\
\text { Pruesapan var. fissisepala } \\
\text { Duyfjes \& Pruesapan }\end{array}$ & 1 & $\begin{array}{l}\text { P. Phonsena, W. de Wilde \& } \\
\text { B. Duyfjes } 4451(\mathrm{~L})\end{array}$ & Thailand, Chiang Mai & HE661370 & HE661531 & HE661449 & - & - & - \\
\hline $\begin{array}{l}\text { Trichosanthes pubera Blume } \\
\text { ssp. rubriflos (Cayla) Duyfjes \& } \\
\text { Pruesapan var. fissisepala } \\
\text { Duyfjes \& Pruesapan }\end{array}$ & 2 & K. Pruesapan et al. $56(\mathrm{~L})$ & Thailand, Kanchanaburi & HE661371 & HE661532 & HE661450 & - & - & - \\
\hline $\begin{array}{l}\text { Trichosanthes pubera Blume } \\
\text { ssp. rubriflos (Cayla) Duyfjes } \\
\text { \& Pruesapan var. rubriflos }\end{array}$ & 1 & R. Zhang 1 (M) & $\begin{array}{l}\text { China, cult. South China BG, } \\
\text { Guangzhou }\end{array}$ & HE661372 & DQ536560 & DQ536688 & DQ535819 & DQ536828 & - \\
\hline $\begin{array}{l}\text { Trichosanthes pubera Blume } \\
\text { ssp. rubriflos (Cayla) Duyfjes \& } \\
\text { Pruesapan var. rubriflos }\end{array}$ & 2 & $\begin{array}{l}\text { P. Phonsena, W. de Wilde \& } \\
\text { B. Duyfjes } 3907(\mathrm{~L})\end{array}$ & Thailand, Saraburi & HE661373 & HE661533 & HE661451 & - & - & - \\
\hline $\begin{array}{l}\text { Trichosanthes quinquangulata } \\
\text { A.Gray }\end{array}$ & 1 & $\begin{array}{l}\text { P. Phonsena, W. de Wilde \& } \\
\text { B. Duyfjes } 4416(\mathrm{~L})\end{array}$ & Thailand, Phetchaburi & HE661374 & HE661534 & HE661452 & - & - & - \\
\hline $\begin{array}{l}\text { Trichosanthes quinquangulata } \\
\text { A.Gray }\end{array}$ & 2 & N. Koonthudthod et al. $326(\mathrm{~L})$ & Thailand, Phetchaburi & HE661375 & HE661535 & HE661453 & - & - & - \\
\hline $\begin{array}{l}\text { Trichosanthes quinquefolia } \\
\text { C.Y.Wu }\end{array}$ & & K. Nanthavong et al. BT 705 (L) & Laos, Khammouan & HE661376 & HE661536 & HE661454 & - & - & - \\
\hline $\begin{array}{l}\text { Trichosanthes reticulinervis } \\
\text { C.Y.Wu ex S.K.Chen }\end{array}$ & & X.F. Deng 131 (IBSC) & China, Guangdong & HE661377 & DQ536605 & DQ536744 & DQ535857 & DQ536876 & DQ536876 \\
\hline $\begin{array}{l}\text { Trichosanthes rosthornii } \\
\text { Harms }\end{array}$ & 1 & Jingliang Chuan 5654 (KUN) & China & HE661378 & HE661537 & HE661455 & - & - & - \\
\hline $\begin{array}{l}\text { Trichosanthes rosthornii } \\
\text { Harms }\end{array}$ & 2 & A. Henry 1626 (LE) & China, Hubei & HE661379 & HE661538 & - & - & - & - \\
\hline $\begin{array}{l}\text { Trichosanthes schlechteri } \\
\text { Harms }\end{array}$ & & W. Takeuchi \& D. Ama 15663 (LAE) & Papua New Guinea & HE661380 & EU155619 & EU155610 & EU155605 & EU155628 & EU155638 \\
\hline
\end{tabular}


Table 1 Voucher information and GenBank accession numbers (Continued)

\begin{tabular}{|c|c|c|c|c|c|c|c|c|c|}
\hline \multicolumn{10}{|l|}{$\begin{array}{l}\text { Trichosanthes sepilokensis } \\
\text { Rugayah }\end{array}$} \\
\hline $\begin{array}{l}\text { Trichosanthes smilacifolia } \\
\text { C.Y.Wu }\end{array}$ & & Qiwu Wang 85620 (KUN) & China & HE661382 & HE661540 & - & - & - & - \\
\hline $\begin{array}{l}\text { Trichosanthes subvelutina } \\
\text { F.Muell. ex Cogn. }\end{array}$ & 1 & I. Telford 9778 (CANB) & Australia, Queensland & HE661383 & HE661541 & HE661456 & - & - & - \\
\hline $\begin{array}{l}\text { Trichosanthes subvelutina } \\
\text { F.Muell. ex Cogn. }\end{array}$ & 2 & F. Davies 1541 (CANB) & Australia, Queensland & HE661384 & HE661542 & HE661457 & - & - & - \\
\hline $\begin{array}{l}\text { Trichosanthes subvelutina } \\
\text { F.Muell. ex Cogn. }\end{array}$ & 3 & N. Nicholson 3110 (BRI) & Australia, New South Wales & HE661385 & HE661543 & HE661458 & - & - & - \\
\hline $\begin{array}{l}\text { Trichosanthes tricuspidata } \\
\text { Lour spp. javanica Pruesapan } \\
\text { \& Duyfjes }\end{array}$ & & $\begin{array}{l}\text { P. Phonsena, W. de Wilde \& } \\
\text { B. Duyfjes } 4414(\mathrm{~L})\end{array}$ & Thailand, Phetchaburi & - & HE661592 & HE661591 & - & - & - \\
\hline $\begin{array}{l}\text { Trichosanthes tricuspidata } \\
\text { Lour. ssp. tricuspidata }\end{array}$ & & $\begin{array}{l}\text { P. Phonsena, W. de Wilde \& } \\
\text { B. Duyfjes } 4007(\mathrm{~L})\end{array}$ & Thailand, Nakhon Sawan & HE661386 & HE661544 & HE661459 & - & - & - \\
\hline $\begin{array}{l}\text { Trichosanthes truncata } \\
\text { C.B.Clarke }\end{array}$ & 1 & $\begin{array}{l}\text { P. Phonsena, W. de Wilde \& } \\
\text { B. Duyfjes } 3917(\mathrm{~L})\end{array}$ & Thailand, Chiang Mai & HE661387 & HE661545 & HE661460 & - & - & - \\
\hline $\begin{array}{l}\text { Trichosanthes truncata } \\
\text { C.B.Clarke }\end{array}$ & 2 & $\begin{array}{l}\text { P. Phonsena, W. de Wilde \& } \\
\text { B. Duyfjes } 4490(\mathrm{~L})\end{array}$ & Thailand, Chiang Mai & HE661388 & HE661546 & HE661461 & - & - & - \\
\hline $\begin{array}{l}\text { Trichosanthes truncata } \\
\text { C.B.Clarke }\end{array}$ & 3 & $\begin{array}{l}\text { P. Phonsena, W. de Wilde \& } \\
\text { B. Duyfjes } 6329(\mathrm{~L})\end{array}$ & Thailand, Chiang Mai & HE661389 & HE661547 & HE661462 & - & - & - \\
\hline Trichosanthes villosa Blume & 1 & $\begin{array}{l}\text { P. Phonsena, W. de Wilde \& } \\
\text { B. Duyfjes } 4669(\mathrm{~L})\end{array}$ & Thailand, Chiang Mai & - & EU037006 & EU037007 & EU037005 & EU037009 & EU037008 \\
\hline Trichosanthes villosa Blume & 2 & $\begin{array}{l}\text { P. Phonsena, W. de Wilde \& } \\
\text { B. Duyfjes } 6331(\mathrm{~L})\end{array}$ & Thailand, Chiang Mai & HE661390 & : HE661548 & HE661463 & - & - & - \\
\hline Trichosanthes villosa Blume & 3 & $\begin{array}{l}\text { P. Phonsena, W. de Wilde \& } \\
\text { B. Duyfjes } 4449(\mathrm{~L})\end{array}$ & Thailand, Chiang Mai & HE661391 & HE661549 & HE661464 & - & - & - \\
\hline Trichosanthes villosa Blume & 4 & $\begin{array}{l}\text { P. Phonsena, W. de Wilde \& } \\
\text { B. Duyfjes } 4000(\mathrm{~L})\end{array}$ & Thailand, Phetchaburi & HE661392 & HE661550 & - & - & - & - \\
\hline Trichosanthes villosa Blume & 5 & K. Pruesapan et al. 60 (L) & Thailand, Kanchanaburi & HE661393 & HE661551 & HE661465 & - & - & - \\
\hline $\begin{array}{l}\text { Trichosanthes fissibracteata } \\
\text { C.Y.Wu ex C.Y.Cheng \& Yueh }\end{array}$ & & Shaowen Yu 974 (KUN) & China, Yunnan & HE661394 & HE661552 & HE661466 & - & - & - \\
\hline $\begin{array}{l}\text { Trichosanthes wallichiana } \\
\text { (Ser.) Wight }\end{array}$ & & A. Henry 9432 (LE) & China, Yunnan & HE661395 & HE661553 & - & - & - & - \\
\hline Trichosanthes wawrae Cogn. & & B. Gravendeel et al. 631 (L) & Indonesia, Java & HE661396 & HE661554 & HE661467 & - & - & - \\
\hline
\end{tabular}


Polymerase chain reaction (PCR) amplification of purified total DNA was performed in $200 \mu \mathrm{l}$ reaction tubes with a total volume of $50 \mu \mathrm{l}$. Each tube contained a mixture of $5 \mu \mathrm{l}$ reaction buffer (ABgene, 10x), $3 \mu \mathrm{l} \mathrm{MgCl} 2$ $(25 \mathrm{mM}), 1 \mu \mathrm{l}$ dNTP's $(10 \mu \mathrm{M}), 0.25 \mu \mathrm{l}$ Taq-polymerase

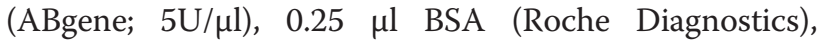
$12.5 \mu \mathrm{l}$ of each primer $(2 \mathrm{mM}), 14.5 \mu \mathrm{l}$ Milli-Q water and $1 \mu \mathrm{l}$ template DNA. The ITS region was amplified using the primer pair ITS-P17 and ITS-26 S-82R [46] with the following PCR protocol $97^{\circ} \mathrm{C} 5 \mathrm{~min}$., $\left(97^{\circ} \mathrm{C}\right.$ 30 s., $55^{\circ} \mathrm{C} 1 \mathrm{~min}$., $72^{\circ} \mathrm{C} 1 \mathrm{~min}$.) $x 35,72^{\circ} \mathrm{C} 10 \mathrm{~min} ., 4^{\circ} \mathrm{C} \infty$; matK with primers matK-2.1a [47] and matK-1440R [48], $95^{\circ} 5$ min., (95 30 s., $50^{\circ} 1$ min., $72^{\circ} 1$ min.) $\times 35,72^{\circ}$ $10 \mathrm{~min} .4^{\circ} \infty$; and rpl20-rps 12 using the primers rpl20 and rps12 [49], $95^{\circ} 5 \mathrm{~min}$. , $^{\circ} 35^{\circ} 30 \mathrm{s.}, 53^{\circ} 1 \mathrm{~min}$., $72^{\circ}$ $1 \mathrm{~min}$.) $x 35,72^{\circ} 10 \mathrm{~min}$., $4^{\circ} \infty$. Sequencing was performed by Macrogen Inc. (Seoul, South Korea) on an ABI3730XL automated sequencer (Applied Biosystems). The same primers as used in the PCR were used for the sequencing reactions.

\section{Sequence alignment}

Sequence trace files were compiled into contigs with the program Gap4 and edited using Pregap4 [50], both part of the Staden package [51]. Sequences were aligned manually in Se-Al [52]. The final matrix included rpl20rps12 (100\% of taxa), ITS (96\%), matK (84\%), trnL-F spacer $(31 \%)$, trnL intron $(28 \%)$, and $r b c L(20 \%)$. The three latter regions increased statistical support values at early-branching clades. Sequences were concatenated, and gap-coded using the Simmons and Ochoterena simple method [53] implemented in SeqState [54].

\section{Phylogenetic analyses}

Selection of best-fit models of nucleotide substitution for the nuclear and plastid data partitions relied on the Akaike Information Criterion (AIC and AICc) as implemented in JModelTest version 0.1.1 [55,56]. Likelihood calculations were carried out for 88 substitution models on an ML-optimized tree. The best-fitting model for the combined data was the general time-reversible (GTR) model, with a proportion of invariable sites (I) and rate variation among sites $(\mathrm{G})$ with four rate categories. Maximum likelihood tree searches and bootstrapping of the combined data (using 1000 replicates) relied on RAxML version 7.2.6 [57] on the CIPRES cluster [58].

Bayesian tree searching used MrBayes [59] on the CIPRES cluster [58]. The combined data were analyzed using three partitions (nuclear, plastid, gap data), allowing partition models to vary by unlinking gamma shapes, transition matrices, and proportions of invariable sites. Markov chain Monte Carlo (MCMC) runs started from independent random trees, were repeated twice, and extended for 10 million generations, with trees sampled every 1000th generation. We used the default priors in MrBayes, namely a flat Dirichlet prior for the relative nucleotide frequencies and rate parameters, a discrete uniform prior for topologies, and an exponential distribution (mean 1.0) for the gamma-shape parameter and branch lengths. Convergence was assessed by checking that the standard deviations of split frequencies were $<0.01$; that the $\log$ probabilities of the data given the parameter values fluctuated within narrow limits; that the convergence diagnostic (the potential scale reduction factor given by MrBayes) approached one; and by examining the plot provided by MrBayes of the generation number versus the log probability of the data. Trees saved prior to convergence were discarded as burn-in (10 000 trees) and a consensus tree was constructed from the remaining trees.

The data matrix and trees have been deposited in TreeBASE (www.treebase.org; study number 12339).

\section{Divergence time estimation}

Divergence times were estimated using the Bayesian relaxed clock approach implemented in BEAST version 1.6.2 [60]. Searches used a Yule tree prior, the GTR + G substitution model, and 50 million MCMC generations, sampling every 1000th generation. Six monophyletic groups were defined based on the results of our phylogenetic analyses and previously published phylogenies $[18,20,44]$. Tracer version 1.5 [61] was used to check that effective sampling sizes had all reached $>200$, suggesting convergence of the chains. TreeAnnotator, part of the BEAST package, was then used to create a maximum clade credibility tree, with the mean divergence ages shown for all nodes with $>95 \%$ highest posterior density.

Calibration relied on Cucurbitaceae fossils assigned to particular nodes (labeled A--C in Figure 3), using a gamma prior distribution with the fossil age as the offset and shape and scale parameter chosen to add a 95\% CI of c. 10 Ma older than the fossil. (A) The root node, that is, the most recent common ancestor of Momordica and Trichosanthes, was constrained to $55.8 \mathrm{Ma}$ with a shape parameter of 1.0 and a scale of 1.0, based on seeds from the Paleocene/Eocene Felpham flora representing the oldest Cucurbitaceae and dated to c. $55.8 \mathrm{Ma}$ [62]. (B) The crown node of the Trichosanthes/Gymnopetalum clade was constrained to $34 \mathrm{Ma}$ with a shape parameter of 1.0 and a scale of 3.4, based on Trichosanthes seeds from the Upper Eocene of Bulgaria [25] dated to c. $34 \mathrm{Ma}$ and seeds from the Oligocene of West Siberia [26] dated to c. $23.8 \mathrm{Ma}$ [27]. (C) The divergence of Marah and Echinocystis was set to $16 \mathrm{Ma}$ with a shape parameter of 1.0 and a scale of 3.35 , based on leaves and a fruit representing Marah from the Miocene of Stewart Valley, Nevada (M. Guilliams and D. M. 
Erwin, University of California, Berkeley, in preparation; the fruit comes from the Fingerrock Wash site, dated to c. $16 \mathrm{Ma}$, the leaf from the Savage Canyon Formation, dated to c. $14.5 \mathrm{Ma}$ ). Absolute ages were taken from the geologic time scale of Walker and Geissman [63]. We also tested lognormal and exponential prior distributions, which gave very similar age estimates (results not shown).

\section{Biogeographical analysis}

Biogeographic reconstruction relied on statistical dispersalvicariance analysis using S-DIVA version 2.0 [64] as implemented in RASP, which carries out parsimony inference on the chain of trees obtained from an MCMC search [65,66], in our case the 8000 post burn-in Bayesian trees resulting from the BEAST dating analysis. $S$-DIVA averages the frequencies of an ancestral range at a node in ancestral reconstructions over all trees, with alternative ancestral ranges at a node weighted by the frequency of the node [64]. Range information for all species was compiled from taxonomic treatments $[9,11,13-16]$, and the coded distribution areas were: A) Australia and New Guinea, B) Wallacea, C) Insular Sunda Malesia, D) Mainland Southeast Asia, E) India and adjacent countries, F) Africa, Europe and the New World.

\section{Authors' contributions}

$\mathrm{HB}$ conceived the study, carried out the molecular genetic analyses, and drafted the manuscript. HS participated in the design of the study and data analysis, and also contributed field observations. SR and MT participated in the design and coordination of the study, and SR also helped with clock calibration and writing. All authors read and approved the final manuscript.

\section{Acknowledgments}

We thank W.J. de Wilde and B. Duyfjes for leaf samples, advice on species sampling and taxonomy, and comments on preliminary results; W.E. Cooper, N. Filipowicz, C. Jeffrey, and I. Telford for leaf samples; L. Nauheimer for Figure 3, B. Schlumpberger and A. Kelber for advice on function of petal fringes, M. Guilliams and D.M. Erwin for information on Marah fossils, and curators of the herbaria $A, B R I, C N S, E, G H, K, K U N, K Y O, L, L E, M, M O, P, S$, UC, UPS and US for samples, loans, or help during visits to their institutions. This research was supported by SIDA-SAREC grant SWE-2005-338, Anna Maria Lundins stipendiefond, Helge Ax:son Johnsons stiftelse, Regnells botaniska resestipendium, SYNTHESYS grant GB-TAF-4255, and Knut och Alice Wallenbergs medel till rektors förfogande.

\begin{abstract}
Author details
'Department of Systematic Biology, Uppsala University, Norbyvägen 18 D, Uppsala SE-75236, Sweden. ${ }^{2}$ Harvard University, Department of Organismic and Evolutionary Biology, 22 Divinity Avenue, Cambridge, MA 02138, U.S.A. ${ }^{3}$ Department of Systematic Biology, Uppsala University, Norbyvägen 18 D, Uppsala SE-75236, Sweden. ${ }^{4}$ University of Munich (LMU), Systematic Botany and Mycology, Menzinger Str. 67, Munich 80638, Germany.
\end{abstract}

Received: 10 February 2012 Accepted: 21 June 2012 Published: 3 July 2012

\section{References}

1. Endress PK, Matthews ML: Elaborate petals and staminodes in eudicots: diversity, function, and evolution. Org Divers Evol 2006, 6:257-293.

2. Miyake T, Yamaoka R, Yahara T: Floral scents of hawkmoth-pollinated flowers in Japan. J Plant Res 1998, 111:199-205.
3. Delpino F: Ulteriori osservazioni e considerazioni sulla dicogamia nel regno vegetale. Atti Soc Ital Sci Nat 1870, 13:167-205.

4. Vogel S: Blütenbiologische typen als elemente der Sippengliederung. Jena: G. Fischer; 1954

5. Kelber A, Balkenius A, Warrant EJ: Scotopic colour vision in nocturnal hawkmoths. Nature 2002, 419:922-925.

6. Kelber A, Balkenius A, Warrant EJ: Colour vision in diurnal and nocturnal hawkmoths. Integr Comp Biol 2003, 43:571-579.

7. Schaefer H, Kocyan A, Renner SS: Linnaeosicyos (Cucurbitaceae): a new genus for Trichosanthes amara, the Caribbean sister species of all Sicyeae. Syst Bot 2008, 33:349-355.

8. Schaefer H, Renner SS: Cucurbitaceae. In The families and genera of vascular plants. 10th edition. Berlin: Springer; 2011:112-174.

9. de Wilde WJJO, Duyfjes BEE: Cucurbitaceae. Leiden: Foundation Flora Malesiana; 2010:19.

10. Perry LM, Metzger J: Medicinal plants of East and Southeast Asia: attributed properties and uses. MA: MIT press Cambridge; 1980.

11. Chakravarty HL: Monograph on Indian Cucurbitaceae (Taxonomy and Distribution). Rec Bot Surv India 1959, 27:28-56.

12. Telford IR: Cucurbitaceae. In Flora of Australia, Volume 8. Volume 8th edition. Edited by Flora of Australia Editorial Committee. Canberra: Australian Govt. Pub. Service; 1982:194-198.

13. Ohba H: Cucurbitaceae. In Flora of Japan. Washington: Smithsonian Institution; 1984

14. Duyfjes BEE, Pruesapan K: The genus Trichosanthes L. (Cucurbitaceae) in Thailand. Thai For Bull (Bot) 2004, 32:76-109.

15. Lu A-M, Huang L-Q, Chen S-K, Jeffrey C: Cucurbitaceae. In Flora of China. Vol. 19 (Cucurbitaceae through Valerianaceae, with Annonaceae and Berberidaceae). St. Louis: Missouri Botanical Gardens Press; 2011.

16. Cooper WE, de Boer HJ: A taxonomic revision of Trichosanthes L. (Cucurbitaceae) in Australia, including one new species from the Northern Territory. Austrobaileya 2011, 8:364-386.

17. de Boer HJ, Thulin M: Synopsis of Trichosanthes (Cucurbitaceae) based on recent molecular phylogenetic data. PhytoKeys 2012, 12:23-33.

18. Schaefer H, Renner SS: Phylogenetic relationships in the order Cucurbitales and a new classification of the gourd family (Cucurbitaceae). Taxon 2011, 60:122-138.

19. de Wilde WJJO, Duyfjes BEE: Review of the Genus Gymnopetalum (Cucurbitaceae). Blumea 2006, 51:281-296.

20. Schaefer H, Heibl C, Renner SS: Gourds afloat: a dated phylogeny reveals an Asian origin of the gourd family (Cucurbitaceae) and numerous oversea dispersal events. Proc R Soc Lond B 2009, 276:843-851.

21. Rugayah M, de Wilde WJJO: Conspectus of Trichosanthes (Cucurbitaceae) in Malesia. Reinwardtia 1999, 11:227-280.

22. de Wilde WJJO, Duyfjes BEE: The genus Trichosanthes (Cucurbitaceae) in Sabah. Sandakania 2004, 14:5-32.

23. Yueh $\mathrm{CH}$, Cheng $\mathrm{CY}$ : A preliminary study of the Chinese medicinal species of genus Trichosanthes L. Act Phytotax Sin 1974, 12:415-458.

24. Cogniaux CA: Cucurbitaceae. In Monographiae Phanerogamarum Prodromi nunc Continuato, nunc Revisio Auctoribus Alphonso et Casimir de Candolle Aliisque Botanicis Ultra Memoratis. 3rd edition. Paris; 1881:325-951.

25. Palamarev E: Die Eozane Flora des Burgas-Beckens. Izvestiya na Botaniceskiya Institut 1973, 24:75-124.

26. Dorofeev PI: Tretichnyye Flory Zapadnoy Sibiri. [The Tertiary Floras of Western Siberia]. Moskva-Leningrad: Izd Akad Nauk SSSR; 1963.

27. Gnibidenko Z, Semakov N: Paleomagnetism of boundary oligocenemiocene deposits in the Kompasskii Bor tract on the Tym River (Western Siberia). Izvestiya Phys Solid Earth 2009, 45:70-79.

28. Reid E: Recherches sur quelques graines pliocènes du Pont-du-Gail (Cantal). Bull Soc Geol France 1920, 20:48-87.

29. Szafer W: Flora pliocenska z Kroscienka nad Dunajcem. Pol Akad Umiej Rozpr Wydz Mat-Przyr, Dziat B, Nauxi Biol 1946, 72:1-213 [177-178].

30. Mai $D$, Walther H: Die Pliozänen Floren von Thüringen, Deutsche Demokratische Republik. Quartärpaläontologie 1988, 7:55-297.

31. Kvaček Z, Teodoridis V, Gregor H: The Pliocene Leaf Flora of Auenheim, Northern Alsace (France). Documenta Naturae 2008, 155:1-108.

32. Wilde $V$, Kvaček Z, Bogner J: Fossil leaves of the Araceae from the European Eocene and notes on other aroid fossils. Int J PI Sci 2005, 166:157-183.

33. Hall R: Southeast Asia's changing palaeogeography. Blumea 2009, 54:148-161 
34. Hall R: The plate tectonics of Cenozoic SE Asia and the distribution of land and sea. In Biogeography and geological evolution of SE Asia. Leiden: Backhuys; 1998:99-131

35. Morley R: Palynological evidence for Tertiary plant dispersals in the SE Asian region in relation to plate tectonics and climate. In Biogeography and Geological Evolution of SE Asia. Leiden: Backhuys; 1998:211-234.

36. Voris HK: Maps of Pleistocene sea levels in Southeast Asia: shorelines, river systems and time durations. J Biogeogr 2000, 27:1153-1167.

37. Lohman DJ, de Bruyn M, Page T, von Rintelen K, Hall R, Ng PKL, Shih HT, Carvalho GR, von Rintelen T: Beyond Wallace's line: Genetics and geology inform biogeographic insights in the Indo-Australian archipelago. Annu Rev Ecol Evol Syst 2011, 42:205-226.

38. Simpson GG: Too many lines; the limits of the Oriental and Australian zoogeographic regions. Proc Am Philos Soc 1977, 121:107-120.

39. Kelber A: Pattern discrimination in a hawkmoth: innate preferences, learning performance and ecology. Proc R Soc Lond B 2002, 269:2573-2577.

40. Herrera CM: Selection on complexity of corolla outline in a hawkmothpollinated violet. Evol Trend Plant 1993, 7:9-13.

41. Warren J, James P: Do flowers wave to attract pollinators? A case study with Silene maritima. J Evol Biol 2008, 21:1024-1029.

42. Keraudren-Aymonin M: Cucurbitacées. Paris: Muséum National D'Histoire Naturelle; 1975:15.

43. Swensen SM, Luthi JN, Rieseberg LH: Datiscaceae revisited: monophyly and the sequence of breeding system evolution. Syst Bot 1998, 23:157-169.

44. Kocyan A, Zhang LB, Schaefer H, Renner SS: A multi-locus chloroplast phylogeny for the Cucurbitaceae and its implications for character evolution and classification. Mol Phylogenet Evol 2007, 44:553-577.

45. Yoon CS, Glawe A, Shaw PD: A method for rapid small-scale preparation of fungal DNA. Mycologia 1991, 83:835-838.

46. Popp M, Oxelman B: Inferring the history of the polyploid Silene aegaea (Caryophyllaceae) using plastid and homoeologous nuclear DNA sequences. Mol Phylogenet Evol 2001, 20:474-481.

47. Royal Botanic Gardens Kew, DNA Barcoding. http://www.kew.org/barcoding/ protocols.html.

48. Fior S, Karis PO, Casazza G, Minuto L, Sala F: Molecular phylogeny of the Caryophyllaceae (Caryophyllales) inferred from chloroplast matK and nuclear rDNA ITS sequences. Am J Bot 2006, 93:399.

49. Hamilton MB: Four primer pairs for the amplification of chloroplast intergenic regions with intraspecific variation. Mol Ecol 1999, 8:521-523.

50. Bonfield JK, Smith KF, Staden R: A new DNA sequence assembly program. Nucleic Acids Res 1995, 23:4992.

51. Staden R: The Staden sequence analysis package. Mol Biotechnol 1996, 5:233-241.

52. Rambaut A: Se-Al: sequence alignment editor. v 2.0. Oxford: University of Oxford; 1996.

53. Simmons MP, Ochoterena H: Gaps as characters in sequence-based phylogenetic analyses. Syst Biol 2000, 49:369-381.

54. Muller K: SeqState: primer design and sequence statistics for phylogenetic DNA datasets. App/ Bioinformatics 2005, 4:65-69.

55. Guindon S, Gascuel O: A simple, fast, and accurate algorithm to estimate large phylogenies by maximum likelihood. Syst Biol 2003, 52:696.

56. Posada D: jModelTest: phylogenetic model averaging. Mol Biol Evol 2008, 25:1253.

57. Stamatakis A, Hoover $P$, Rougemont J: A rapid bootstrap algorithm for the RAxML web servers. Syst Biol 2008, 57:758.

58. Miller M, Holder MT, Vos R, Midford P, Liebowitz T, Chan L, Hoover P, Warnow T: The CIPRES portals. CIPRES.; 2010. Website http://www.phylo.org/ subsections/portal. Accessed 06 January 2010.

59. Huelsenbeck JP, Ronquist F: MRBAYES: Bayesian inference of phylogenetic trees. Bioinformatics 2001, 17:754-755

60. Drummond AJ, Rambaut A: BEAST: Bayesian evolutionary analysis by sampling trees. BMC Evol Biol 2007, 7:214

61. Rambaut A, Drummond AJ: Tracer. MCMC Trace Analysis Tool. v 1.5. Oxford: University of Oxford; 2009.

62. Collinson ME, Boulter MC, Holmes PL: Magnoliophyta ("Angiospermae"). In The Fossil Record. London: Chapman and Hall; 1993:809-841.

63. Walker J, Geissman J: 2009 Geologic Time Scale. GSA Today 2009, 19:60-61.
64. Yu Y, Harris AJ, He X: S-DIVA (Statistical Dispersal-Vicariance Analysis): a tool for inferring biogeographic histories. Mol Phylogenet Evol 2010, 56:848-850

65. Nylander JA, Olsson U, Alström P, Sanmartín I: Accounting for phylogenetic uncertainty in biogeography: a Bayesian approach to dispersalvicariance analysis of the thrushes (Aves: Turdus). Syst Biol 2008 57:257.

66. Harris AJ, Xiang QYJ: Estimating ancestral distributions of lineages with uncertain sister groups: a statistical approach to Dispersal-Vicariance Analysis and a case using Aesculus L. (Sapindaceae) including fossils. J Syst Evol 2009, 47:349-368.

doi:10.1186/1471-2148-12-108

Cite this article as: de Boer et al.: Evolution and loss of long-fringed petals: a case study using a dated phylogeny of the snake gourds, Trichosanthes (Cucurbitaceae). BMC Evolutionary Biology 2012 12:108.

\section{Submit your next manuscript to BioMed Central and take full advantage of:}

- Convenient online submission

- Thorough peer review

- No space constraints or color figure charges

- Immediate publication on acceptance

- Inclusion in PubMed, CAS, Scopus and Google Scholar

- Research which is freely available for redistribution

Submit your manuscript at www.biomedcentral.com/submit
C Biomed Central 